\title{
Dopamine Uptake Changes Associated with Cocaine Self-Administration
}

\author{
Erik B Oleson', Sanjay Talluri², Steven R Childers', James E Smith', David CS Roberts', Keith D Bonin² and \\ Evgeny A Budygin*,1
}

'Department of Physiology and Pharmacology, Wake Forest University School of Medicine, Winston-Salem, NC, USA; ${ }^{2}$ Department of Physics, Wake Forest University, Winston-Salem, NC, USA

\begin{abstract}
The present study was designed to reveal the relationship between cocaine-induced dopamine uptake changes and patterns of cocaine self-administration observed under a fixed-ratio schedule. Cocaine was intravenously infused into anesthetized rats, according to interinfusion intervals obtained from self-administering animals, and dopamine uptake changes (apparent $K_{m}$ ) were assessed in the nucleus accumbens using voltammetry. The data demonstrate that cocaine-induced dopamine transporter (DAT) inhibition accounts for the accumbal dopamine fluctuations, which are associated with the cyclic regularity of cocaine intake observed during self-administration. Specifically, the inter-infusion intervals that are maintained during cocaine self-administration correlate with the maintenance of a rapidly changing level of dopamine uptake inhibition, which appears to be tightly regulated. Furthermore, this maintained level of dopamine uptake inhibition was found to shift upward using intervals from animals that had shown an escalation in the rate of cocaine selfadministration. Although no significant change in the apparent $K_{m}$ was revealed in animals that exhibited an escalation in the rate of cocaine intake, an increased dopamine uptake rate was found suggesting an upregulation of DAT number in response to a history of high cocaine intake. This is the first demonstration of the tight correlation that exists between the level of dopamine uptake inhibition and rates of cocaine self-administration. Moreover, a new mathematical model was created that quantitatively describes the changes in cocaine-induced dopamine uptake and correctly predicts the level of dopamine uptake inhibition. This model permits a computational interpretation of cocaine-induced dopamine uptake changes during cocaine self-administration.

Neuropsychopharmacology (2009) 34, I I74-I | 84; doi:10.1038/npp.2008. I86; published online I5 October 2008
\end{abstract}

Keywords: dopamine transporter; addiction; psychostimulants; pharmacokinetics; Michaelis-Menten kinetics; tolerance

\section{INTRODUCTION}

It is commonly accepted that dopamine neurotransmission is essential for the stimulating, reinforcing, and addictive effects of cocaine and other abused drugs (Koob and Bloom, 1988; Volkow et al, 2004). Acutely administered cocaine enhances extracellular dopamine concentrations in specific brain regions, including the caudate putamen and nucleus accumbens (Di Chiara and Imperato, 1988). The magnitudes of cocaine-induced psychomotor activation are positively and highly correlated with dopamine responses detected in these areas (Sabeti et al, 2002; Budygin, 2007). Moreover, subsecond dopamine fluctuations in the nucleus accumbens are associated with cocaine-seeking behavior (Phillips et al, 2003; Stuber et al, 2005).

Levels of extracellular dopamine in the nucleus accumbens appear to regulate the rate of cocaine intake. Early

*Correspondence: Dr EA Budygin, Department of Physiology and Pharmacology, Wake Forest University School of Medicine, Medical Center Blvd, Winston-Salem, NC 27I57, USA, Tel: + I 336 716-8530, Fax: + I 336 716-850।, E-mail: ebudygin@wfubmc.edu

Received 16 May 2008; revised 16 September 2008; accepted 16 September 2008 studies showed that cocaine infusions are self-administered at regular intervals and that the inter-injection interval depends on the unit injection dose (Pickens and Thompson, 1968; Wilson et al, 1971). The timing of this behavior was initially suggested to be associated with fluctuating blood or brain levels of cocaine (Yokel and Piekens, 1974; Gerber and Wise, 1989) with responding being initiated when cocaine levels fall below a threshold level. This idea was extended to include brain dopamine levels by Justice and colleagues who used microdialysis to show that extracellular dopamine rapidly increases following each cocaine injection and drugseeking appears to be initiated when dopamine levels decline to some critical concentration (Pettit and Justice, 1989; Wise et al, 1995). This level has been variously called a trigger point (Wise et al, 1995), set point (Ahmed and Koob, 1998), or satiety threshold (Tsibulsky and Norman, 1999).

Although it is commonly believed that the inhibition of the dopamine transporter (DAT) by cocaine is the mechanism responsible for the elevation of extracellular dopamine in the nucleus accumbens (Wu et al, 2001; Garris and Rebec, 2002; Budygin, 2007); it remains unclear to what extent cocaine-induced DAT inhibition is involved 
in the timing of inter-infusion intervals or whether other mechanisms are involved. For example, subsecond dopamine release (ie dopamine transients) detected when an animal approaches a cocaine-paired lever (Phillips et al, 2003; Stuber et al, 2005) could be important in cocaine selfadministration. Moreover, the onset and time course of cocaine-induced dopamine uptake inhibition observed in some studies (Kiyatkin et al, 2000; Wakazono and Kiyatkin, 2008) are too slow and gradual to account for the rapid fluctuations in dopamine observed during cocaine selfadministration. The fact that rats self-administer cocaine during the peak of dopamine uptake inhibition (Kiyatkin et al, 2000) is inconsistent with the hypothesis that decreases in DAT inhibition trigger responding. However, dopamine uptake inhibition has not been investigated during an actual time course of cocaine self-administration.

In the present study we sought to establish the time course of cocaine-induced dopamine uptake inhibition by using fast-scan cyclic voltammetry to assess changes in dopamine with high temporal resolution (milliseconds) following electrical stimulation. Measurements were taken every $1 \mathrm{~min}$ to allow dopamine uptake inhibition to be evaluated on a time scale relevant to cocaine self-administration. Specifically, dopamine uptake was assessed in the nucleus accumbens of anesthetized rats using infusion rates obtained from two distinct self-administration procedures, one of which produces stable responding across sessions, and another of which results in an escalation of the rate of cocaine intake over a 2-week period (Ahmed and Koob, 1998). A new mathematical model is proposed that accurately describes and predicts the changes of cocaineinduced dopamine uptake inhibition as they occur after cocaine administration.

\section{MATERIALS AND METHODS}

\section{Inter-Infusion Interval Determinations}

These experiments were designed to allow in vivo voltammetric recordings to be performed while i.v. cocaine infusions were being administered in accordance with rates and patterns of responding observed during cocaine selfadministration. The access conditions under which animals self-administer cocaine can change the rate at which infusions are self-administered. In the present study, two access conditions were of interest. Under short-access conditions ( $2 \mathrm{~h}$ sessions) the rate of responding remains stable over daily sessions. Under long-access conditions $(6 \mathrm{~h}$ sessions) the rate of responding increases, or escalates, over daily sessions (Ahmed and Koob, 1998). For both conditions, rates were analyzed after 14 self-administration sessions had occurred. Rates from a short-access group $(n=6)$ remained stable across sessions; therefore multiple sessions were averaged from the final self-administration sessions (30 total sessions). Rates from a long-access group $(n=8)$ increased across sessions; therefore, only rates from the final day of self-administration were averaged across animals. Inter-infusion intervals were determined by averaging the time in seconds occurring between responses for each animal. Each response was considered separately-so, for example - the inter-infusion interval between response one and two would be different from the inter- infusion interval between response eight and nine. To investigate the effects of a history of long-access to cocaine during self-administration on dopamine uptake, an experimental group $(n=7)$ was given long-access to cocaine (see long-access training) before individual animals were infused with predetermined rates during voltammetric recording. Response rates from the experimental group did not differ from the response rates used to calculate the predetermined long-access inter-infusion intervals $(\mathrm{F}(13,169)=0.75$; NS).

\section{General Cocaine Self-Administration}

Male Sprague-Dawley rats weighing approximately $350 \mathrm{~g}$ at the start of the experiment were used as subjects. Rats were anesthetized by an injection of ketamine $(100 \mathrm{mg} / \mathrm{kg}$, i.p.) and xylazine ( $8 \mathrm{mg} / \mathrm{kg}$, i.p.) and implanted with a chronically indwelling Silastic ${ }^{\circledR}$ cannula (CamCaths, Cambridgeshire, UK) into the right jugular vein. The cannula exited through the skin on the dorsal surface in the region of the scapulae (Roberts and Goeders, 1989). Animals were then individually housed in $30 \times 30 \times 30 \mathrm{~cm}$ operant chambers. Following surgery, a stainless steel protective tether that enclosed Tygon ${ }^{\mathbb{R}}$ tubing was connected to a counterbalanced fluid swivel (Instech Laboratories Inc., Plymouth Meeting, PA, USA) mounted above the chamber. The swivel was connected to an infusion pump (Razel Scientific Instruments Inc., Stamford, CT, USA). The cannulae were flushed daily with heparinized saline to maintain patency. Following a recovery period (3-5 days), animals were given access to a cocaine-paired lever during an acquisition phase. During the acquisition phase, cocaine $(0.75 \mathrm{mg} / \mathrm{kg}$ per infusion) was available on a fixed-ratio 1 schedule of reinforcement. Rats were given access to cocaine in daily training sessions, which terminated after a maximum of 20 infusions or a period of $6 \mathrm{~h}$ had elapsed. An animal was considered to have acquired stable self-administration if 20 injections were self-administered during a single session and the pattern displayed consistent inter-infusion intervals. Upon completion of the acquisition phase animals began the long-access training procedure.

\section{Long-Access Training}

One group of animals $(n=7)$ was used for this experiment. Animals were given access to cocaine $(0.75 \mathrm{mg} / \mathrm{kg}$ per infusion) under a fixed-ratio 1 schedule of reinforcement during daily 6-h session for 14 consecutive days. Under long-access conditions animals increase, or 'escalate,' cocaine intake and the rate of cocaine infusions over 14 days (Ahmed and Koob, 1998, 1999). In the present study, as expected, the long-access training procedure resulted in a significant increase in the rate of responding across sessions $(\mathrm{F}(6,78)=7.32 ; p<0.01)$. Voltammetry was performed $24 \mathrm{~h}$ following the final self-administration session for each individual animal.

\section{Voltammetric Recordings}

All of the voltammetric experiments were performed on anesthetized rats. There were at least three important reasons why this design was chosen $v s$ experiments on freely moving animals which self-administer cocaine. First 
of all, an electrical stimulation that is a necessary part of the procedure would change patterns of cocaine self-administration (Phillips et al, 2003). Second, anesthetized animals permit the application of larger electrical current than can be applied to awake rats. These stimulations permit larger dopamine efflux that allows more accurate evaluation of dopamine uptake changes. Third, the pure pharmacological effect of cocaine without any conditioned influence on dopamine neurotransmission that would be observed during cocaine self-administration in awake animals (Phillips et al, 2003; Stuber et al, 2005) was the main focus of this study. It should also be noted, urethane anesthesia was chosen because it does not alter dopamine uptake dynamics (Garris et al, 2003; Sabeti et al, 2003).

Rats were anesthetized with urethane $(1.5 \mathrm{~g} / \mathrm{kg}$, i.p.) and placed in a stereotaxic frame. A carbon fiber electrode was positioned in the nucleus accumbens core $(\mathrm{AP},+1.3$; $\mathrm{L},+$ $1.3 ; \mathrm{V},-6.6 \mathrm{~mm}$ from bregma) and an $\mathrm{Ag} / \mathrm{AgCl}$ reference electrode was implanted in the contralateral hemisphere. A bipolar stimulating electrode was lowered to the ventral tegmental area ipsilateral to the working electrode at $5.2 \mathrm{~mm}$ posterior and $1.0 \mathrm{~mm}$ lateral to bregma. The stimulating electrode depth was optimized to evoke dopamine release in the nucleus accumbens, which was monitored using a carbon fiber microelectrode. The working electrode was prepared using single carbon fibers (T-650; $6-\mu \mathrm{m}$ diameter, Thornel; Amoco, Greenville, SC), which were pulled and sealed in glass capillaries. For voltammetric recordings, the exposed carbon fiber extended $120 \pm 21 \mu \mathrm{m}$ from glass seal. The reference and carbon fiber electrodes were connected to a head-mounted voltammetric amplifier (UNC Electronics Design Facility, Chapel Hill, NC) and voltammetric recordings were made at the carbon fiber electrode every $100 \mathrm{~ms}$ by applying a triangular waveform $(-0.4$ to $+1.2 \mathrm{~V}, 300 \mathrm{~V} /$ s). The signals had an oxidation peak at $+0.6 \mathrm{~V}$ and a reduction peak at $-0.2 \mathrm{~V} v s \mathrm{Ag} / \mathrm{AgCl}$ reference, identifying the released species as dopamine. Data were digitized (National Instruments, Austin, TX) and stored on a computer. Dopamine release was evoked every $1 \mathrm{~min}$ with electrical stimulations (24 rectangular pulses, $60 \mathrm{~Hz}, 300 \mu \mathrm{A}$, $2 \mathrm{~ms} /$ phase, biphasic) and detected by a carbon fiber electrode. Importantly, with this type of stimulation, physiological dynamics of dopamine release were revealed (Montague et al, 2004). At least four stable stimulations of dopamine were collected, and then either a single $(0.75,1.5$, $3.0 \mathrm{mg} / \mathrm{kg}$, i.v.) or multiple injections $(0.75 \mathrm{mg} / \mathrm{kg}$, i.v.) of cocaine (cocaine hydrochloride obtained from the National Institute on Drug Abuse, Rockville, MD, USA; the drug was dissolved in sterile $0.9 \%$ saline) were administered. The drug was administered as an experimenter-delivered bolus over $4 \mathrm{~s}$ in a volume of $0.10-0.13 \mathrm{ml}$.

Carbon fiber microelectrodes were calibrated with known concentrations of dopamine $(2-5 \mu \mathrm{M})$ in artificial CSF (aCSF) at room temperature. The aCSF consisted of (in $\mathrm{mM}$ ): $\mathrm{NaCl} 126, \mathrm{KCl} 2.5, \mathrm{NaH}_{2} \mathrm{PO}_{4} 1.2, \mathrm{CaCl}_{2} 2.4, \mathrm{MgCl}_{2} 1.2$, $\mathrm{NaHCO}_{3} 25$, glucose 11 and was $\mathrm{pH}$ adjusted to 7.4. Calibrations were carried out in triplicate and the average value for the current at the peak oxidation potential was used to normalize in vivo signals to dopamine concentration. Dopamine uptake was determined from the clearance rate of dopamine following the termination of the stimulus and was assumed to follow Michaelis-Menten kinetics
(Wightman and Zimmerman, 1990; Garris and Rebec, 2002). A detailed description regarding a model characterizing changes in extracellular dopamine concentrations evoked by electrical stimulation as a balance between the opposing mechanisms of release and uptake was provided in previous publications (Wightman et al, 1988; Wightman and Zimmerman, 1990; Wu et al, 2001; Garris and Rebec, 2002). The changes in dopamine during and after electrical stimulation were fit using Equation (1).

$$
\mathrm{d}[\mathrm{DA}] / \mathrm{d} t=(f)[\mathrm{DA}] \mathrm{p}-\left(V_{\max } /\left\{\left(K_{\mathrm{m}} /[\mathrm{DA}]\right)+1\right\}\right)
$$

where $f$ is the stimulation frequency $(\mathrm{Hz}),[\mathrm{DA}] \mathrm{p}$ the concentration of dopamine released per stimulus pulse. $V_{\max }$ is a maximal velocity of dopamine uptake, which is proportional to the number of available DAT proteins. $K_{\mathrm{m}}$ is the substrate (dopamine) concentration at one half of $V_{\max }$. This second uptake parameter $\left(K_{\mathrm{m}}\right)$ is a complex constant, related to the affinity of dopamine for the DAT and its rate of turnover. The baseline value of $K_{\mathrm{m}}$ was taken to be $\approx 0.2 \mu \mathrm{M}$, a value determined in rat brain synaptosomes (Near et al, 1988; Garris and Rebec, 2002). The integral form of the above equation was used to simulate the dopamine response using single curve analysis. This method, which also agrees favorably with other analyses, has proven to be particularly convenient for the evaluation of dopamine uptake changes induced by competitive DAT inhibitors such as cocaine (Wu et al, 2001; Garris and Rebec, 2002; Budygin, 2007). Dopamine signals for each rat were fit individually at all time points before and after cocaine injections. The $K_{\mathrm{m}}$ was fixed and the other variables were determined, when predrug parameters were calculated. The calculation of $K_{\mathrm{m}}$ requires knowledge of $V_{\max }$ and [DA]p and a steady-state response of electrically evoked dopamine concentration ( $\mathrm{Wu}$ et al, 2001). Consequently, when the cocaine effect on the dopamine efflux was modeled, $K_{\mathrm{m}}$ became the main subject of manipulation, whereas $V_{\max }$ was kept close to the predrug value. As a final point of the fitting procedure, $[\mathrm{DA}] \mathrm{p}$ was adjusted to obtain a best fit.

\section{Statistical Analysis}

Data were analyzed in GraphPad Prism (GraphPad Software, San Diego, CA). A $t$-test, one-way repeated-measures and two-way ANOVAs with Bonferroni posttests were used to determine statistical significance. The data are presented as mean \pm SEM and the criterion of significance was set at $p<0.05$. For the mathematical model, which describes cocaine-induced dopamine uptake changes, MATLAB was used to fit the experimental data using a least-squares minimization procedure. The predicted curves were also generated using MATLAB.

\section{RESULTS}

\section{Intravenous Cocaine Dose Dependently Increases Apparent $K_{\mathrm{m}}$ in the Rat Nucleus Accumbens (Experiment 1)}

In this experiment, dopamine uptake inhibition was assessed in naive rats following single i.v. infusions of cocaine. Electrically evoked dopamine concentrations in the nucleus accumbens were stable before drug injections. 
Saline administration $(0.3 \mathrm{ml} /$ inf, i.v. $)$ did not significantly modify dopamine signals over the time course of this experiment (Figure 1a). The administration of single doses of cocaine elicited fast and robust (2- to 4 -fold) increases in extracellular dopamine (Figure 1b). Kinetic analysis of the evoked dopamine signals indicated that the increases were associated with an increase in the apparent $K_{\mathrm{m}}\left(K_{\mathrm{m}}(\mathrm{app})\right)$ for dopamine uptake. This effect reached a maximum within $1 \mathrm{~min}$ following all doses of cocaine and then gradually decayed. Figure 2a shows dose-dependent increases in $K_{\mathrm{m}}(\mathrm{app})$ occurring $1 \mathrm{~min}$ after an i.v. infusion when dopamine uptake inhibition was maximal $(\mathrm{F}(3,16)=52.3 ; p<0.01)$. Bonferroni posttests indicated significant differences in $K_{\mathrm{m}}(\mathrm{app})$ after $3.0 v s 1.5 \mathrm{mg} / \mathrm{kg}$ of cocaine $(p<0.01)$ and after $0 v s 0.75 \mathrm{mg} / \mathrm{kg}(p<0.01)$. There was a trend toward significance between 1.5 and $0.75 \mathrm{mg} / \mathrm{kg}$ $(p=0.056)$. No significant changes in the maximal rate of dopamine uptake $\left(V_{\max }\right)$ were revealed following any cocaine dose (Figure 2b). The predrug value for $V_{\max }$ $(1.79 \pm 0.15 \mu \mathrm{M} / \mathrm{s})$ is consistent with previously published $V_{\max }$ values obtained in the nucleus accumbens core region (Mateo et al, 2004). The amplitude of electrically evoked dopamine release was increased by cocaine $(\mathrm{F}(3,16)=9.1$;

$$
\text { a }
$$

saline
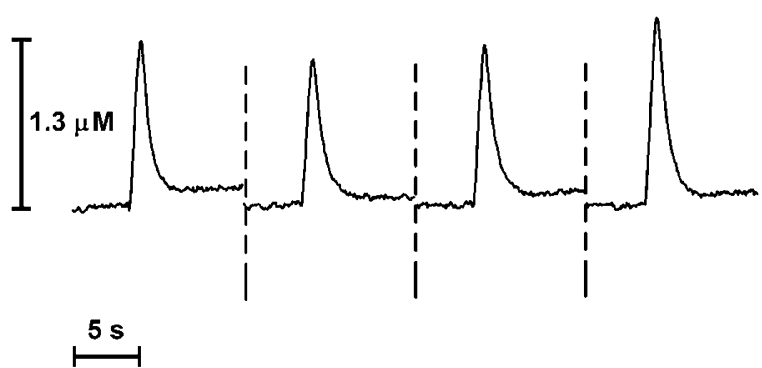

b

cocaine

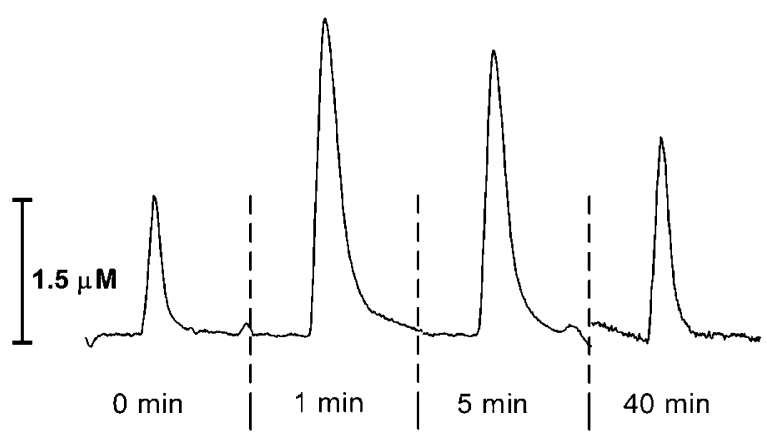

Time after injection

Figure I Representative concentration-time plots of dopamine measured in the rat nucleus accumbens before and following a single i.v. infusion of saline (0.3 ml/inf) (a) and cocaine $(1.5 \mathrm{mg} / \mathrm{kg})$ (b). An infusion of saline did not induce any changes in dopamine peak height or in $K_{m}$ (app). In contrast, cocaine significantly increased dopamine peak height and $K_{m}$ (app). Maximal dopamine peak height occurred within approximately I min after cocaine administration and then gradually decayed ( $1.33 \pm 0.20$ (predrug), $2.82 \pm 0.24$ (I min), $1.74 \pm 0.21$ (5 min), $1.42 \pm 0.13$ (40 min) $\mu \mathrm{M} ; n=5$ ). $K_{\mathrm{m}}$ (app) values were $0.18 \pm 0.01$ (predrug), $1.13 \pm 0.17$ (I min), $0.94 \pm 0.19$ (5 min), $0.43 \pm 0.07$ (40 min) $\mu M ; n=5$. $p<0.05)$ in the same fashion as $K_{\mathrm{m}}(\mathrm{app})$ (Figure 2c). Bonferroni posttests revealed significant differences in this parameter after 1.5 and $3.0 \mathrm{mg} / \mathrm{kg}$ of cocaine $v s$ predrug values $(p<0.05)$. Importantly, electrically evoked dopamine release does not reflect the basal dopamine concentration in rat brain.
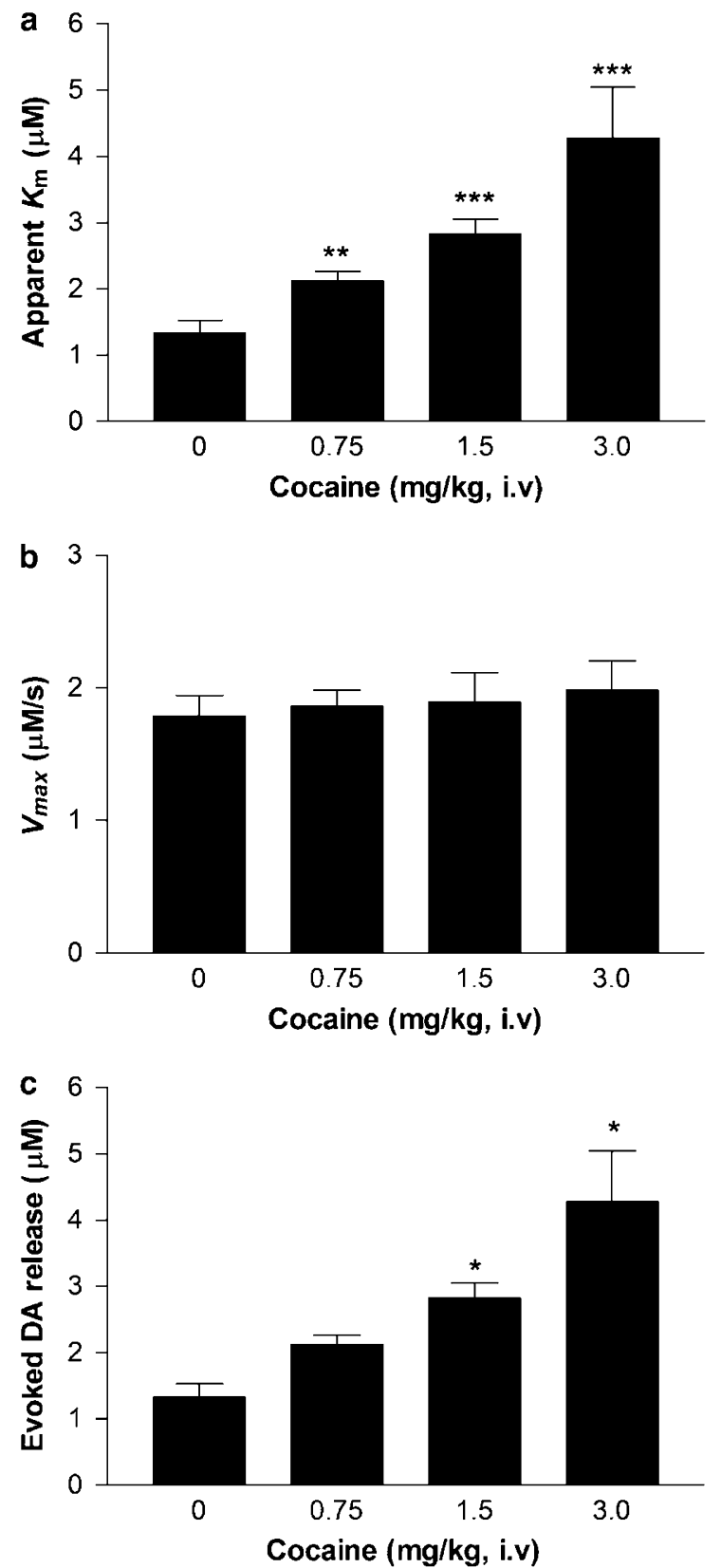

Figure 2 Effect of single cocaine (i.v.) injection on dopamine release and uptake in rat nucleus accumbens. Electrically evoked dopamine concentrations and uptake parameters, reported as an $K_{m}(a p p)$ and $V_{\text {max }}$, were measured I min after a $4 \mathrm{~s}$ cocaine infusion. (a) $K_{m}$ (app) was dose dependently increased following a single infusion of intravenous cocaine $\left(0.75,1.5\right.$, or $3 \mathrm{mg} / \mathrm{kg}$ ). (b) No changes in $V_{\max }$ were detected. (c) Similar to the effect on $K_{m}(\mathrm{app})$, cocaine significantly enhanced electrically evoked dopamine release. Data are means \pm SEM of five rats per group. ${ }^{*} p<0.05$, *** $p<0.005$, and $* * * *<0.001$. 
Two Distinct Phases of Dopamine Uptake Inhibition are Observed When Cocaine is Infused Using Intervals Observed During Self-Administration (Experiment 2)

In this experiment, cocaine $(0.75 \mathrm{mg} / \mathrm{kg})$ was i.v. infused into naive rats using inter-infusion intervals that were predetermined from rats that self-administered cocaine under a fixed-ratio 1 schedule (2-h session) of drug delivery (short-access training) (Figure 3). These intervals were 1.11, $2.18,3.82,4.52,4.47,4.67,4.83,4.70,5.33,5.37$, and $5.18 \mathrm{~min}$. Each cocaine infusion significantly increased $K_{\mathrm{m}}$ (app) compared to the drug pre-infusion value $(\mathrm{F}(18,72)=49.21 ; p<0.01, n=5)$. Following the first four cocaine infusions (loading phase), the level of dopamine uptake inhibition reached a steady-state oscillation that persisted for the duration of the experiment (maintenance phase). During the maintenance phase upper and lower thresholds were discernible. The upper threshold, which is distinguishable as the crests of the fluctuating level of dopamine uptake inhibition, is defined as the average of the maximal values of $K_{\mathrm{m}}(\mathrm{app})$. Likewise, the lower threshold, which is evident as the troughs of dopamine uptake
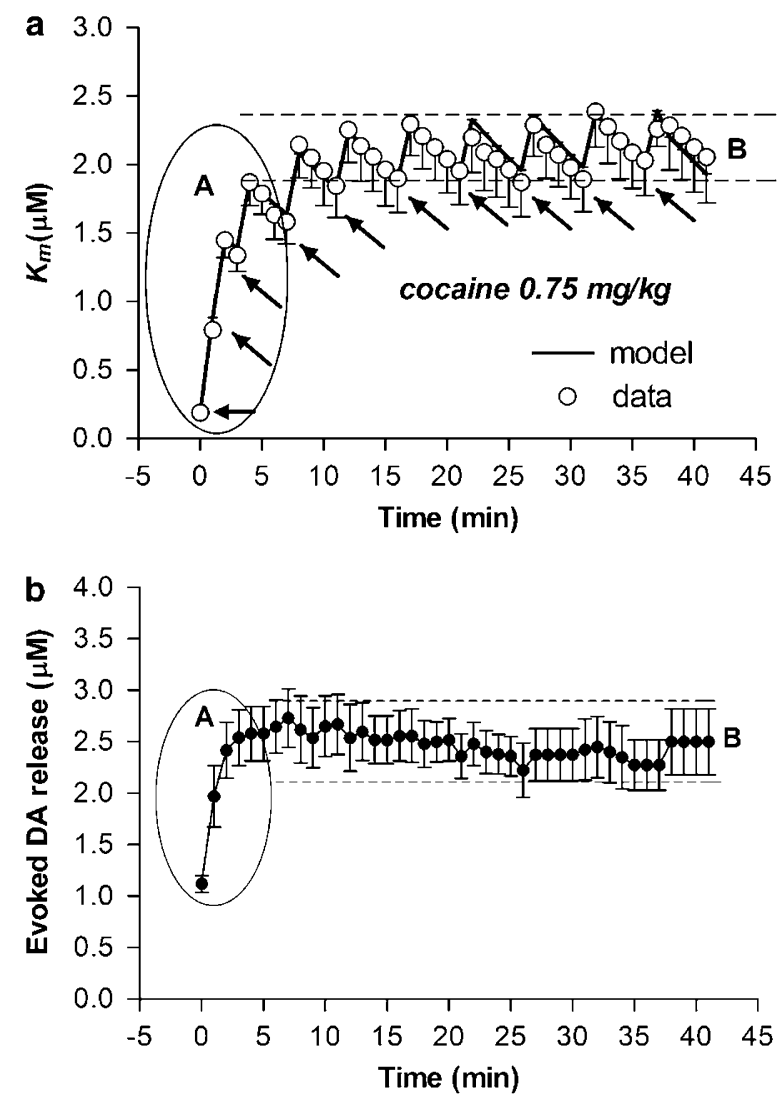

Figure 3 (a) Dopamine uptake changes obtained with inter-infusion intervals predetermined from rats self-administrating cocaine. Dopamine uptake changes, which are measured as alterations in $K_{m}(a p p)$ are indicated by empty circles. The solid line shows modeled changes in dopamine uptake inhibition. The time points when cocaine $(0.75 \mathrm{mg} / \mathrm{kg}$, i.v. $)$ was injected over $4 \mathrm{~s}$ are depicted as arrows. Dopamine uptake changes associated with responding during the loading phase are indicated by the oval (A). A steady-state oscillation of dopamine uptake inhibition that is observed throughout the maintenance phase (B) is indicated by dashed lines. (b) The time course of cocaine on the peak evoked dopamine concentration. Data are means \pm SEM of five rats per group. inhibition, is defined as the average of the minimal values of $K_{\mathrm{m}}(\mathrm{app})$. There was a significant difference between the upper and lower thresholds $(t=4.8 ; p<0.01, n=6$, paired $t$-test).

As expected, the amplitude of electrically evoked dopamine efflux was also significantly increased following cocaine infusions $(\mathrm{F}(41,164)=6.2 ; p<0.01, n=5)$. The time course of the changes in evoked dopamine efflux paralleled the time course of the increase in $K_{\mathrm{m}}(\mathrm{app})$ (Figure 3, inset), suggesting that the effect of cocaine on dopamine peak height is preferentially driven by competitive antagonism of the DAT. However, multiple mechanisms are also involved in the effect of cocaine on electrically evoked dopamine. For example, cocaine can enhance dopamine release by mobilizing a synapsin-dependent reserve pool (Venton et al, 2006). Moreover, electrically evoked dopamine release is also potentially subject to $\mathrm{D}_{2}$ dopamine receptormediated autoinhibition (Schmitz et al, 2001, 2002; Wu et al, 2002), which takes place during cocaine-induced DAT blockade (Grace, 2000). As the dopamine peak amplitude is affected by many factors, the interpretation of cocaine effects on dopamine neurotransmission using this parameter can be complicated.

\section{Dynamics of Dopamine Uptake Inhibition can be Mathematically Modeled and Predicted (Experiment 3)}

A new mathematical model was developed to quantitatively predict the behavior of the apparent Michaelis-Menten constant $K_{\mathrm{m}}(\mathrm{app})$ as a function of time. The model depends on three parameters: the initial cocaine concentration of each injection, the probability of dopamine diffusion and transport in the absence of DAT, and the rate of dopamine removal in the presence of DAT.

This model assumes a very simple form for the apparent Michaelis constant $K_{\mathrm{m}}(\mathrm{app})$ based on the assumption that the DAT acts as though it can be in one of two states or conditions, ie that the DAT is either occupied or unoccupied by cocaine. In this case, the probability that a transporter is occupied by a cocaine molecule is represented by $p_{\mathrm{o}}$. If $p_{\mathrm{u}}$ represents the probability that a transporter is unoccupied by a cocaine molecule, then these two probabilities must sum to 1 , ie $p_{\mathrm{o}}+p_{\mathrm{u}}=1$. This relation applies as the model is binary, and the DAT is either occupied or unoccupied by a cocaine molecule. Cocaine acts as an inhibitor, by attaching to the DAT that prevents a dopamine molecule from attaching to the same transporter. Thus, a physically reasonable model for these probabilities involves the ratio of the number of available inhibitor (cocaine) molecules [I] to the number of accessible DATs [T]. The number of accessible transporters is assumed fixed. The number of available inhibitor molecules is simply proportional to the inhibitor concentration. If this ratio is defined to be

$$
s=\frac{[\mathrm{I}]}{[\mathrm{T}]}
$$

then the probability that cocaine will occupy a transporter is equal to $s$, ie $p_{\mathrm{o}}=s$. This last relation, that $p_{\mathrm{o}}=s$, is only true as long as the number of occupied DATs remains small, ie the occupation probability is much less than 1 . However, 
when the number of available inhibitors becomes large, ie comparable to or higher than the numbers of DATs, then the probability must be modified to account for this moderate to high ratio of inhibitors to transporters. In the case where the transporters are basically saturated with inhibitors, then the occupation probability will reach its maximum value of 1 . An appropriate model for such a system assumes that the inhibitor occupation probability $p_{\mathrm{o}}$ is given by

$$
p_{\mathrm{o}}=\frac{s}{1+s}
$$

As an aid to demonstrating that such a model is reasonable, note that this probability has the correct form in the two extreme cases of low inhibitor-to-transporter ratios $(s \ll 1$, $\left.p_{\mathrm{o}}=s\right)$ and large inhibitor concentration-to-transporter ratios $\left(s \gg 1, p_{\mathrm{o}}=1\right)$.

This inhibitor-occupied transporter probability can be related to the apparent Michaelis constant. In enzyme reaction rate theory (see Voet and Voet, 2004), an apparent $K_{\mathrm{m}}(\mathrm{app})$ in the presence of an inhibitor is given by

$$
K_{\mathrm{m}}(\operatorname{app})=\left(1+\frac{[\mathrm{I}]}{K_{1}}\right) K_{\mathrm{m}}
$$

where $K_{\mathrm{m}}$ is the Michaelis constant in the absence of inhibitor, and the constant $K_{1}$ is the equilibrium constant associated with the enzyme-inhibitor complex. To mimic this standard relation from enzyme reaction rate theory, it is assumed that a Michaelis constant depends on the inhibitor-occupied transporter probability in the following way

$$
K_{\mathrm{m}}(\mathrm{app})=\left(\frac{1}{1-p_{\mathrm{o}}}\right) K_{\mathrm{m}}
$$

The factor multiplying $K_{\mathrm{m}}$ in both of these cases can be related, in the case of small inhibitor concentration, using the geometric series expansion of the prefactor in the last equation. The geometric series $1+p_{\mathrm{o}}+p_{\mathrm{o}}^{2}+\ldots$ sums to the prefactor in Equation (5) above. So if all the small numbers in the geometric series are dropped, such as $p_{\mathrm{o}}^{2}$ and higher powers of $p_{o}$, then for small $p_{o}$ or [I] Equation (5) for $K_{\mathrm{m}}(\mathrm{app})$ can be written:

$$
K_{\mathrm{m}}(\mathrm{app}) \approx\left(1+p_{\mathrm{o}}\right) K_{\mathrm{m}}=\left(1+\frac{[\mathrm{I}]}{K_{1}}\right) K_{\mathrm{m}}
$$

Thus, this model, Equation (5), gives the expected result in the extreme case of low inhibitor numbers (or concentrations),Equation (4). In the extreme case of high inhibitor concentrations the occupation probability $p_{\mathrm{o}}$ approaches 1 , and the effective Michaelis constant approaches infinity (see Equation (5)). This means that inhibitors occupy all the available transporter sites and so the reaction with dopamine shuts down and dopamine is not taken up at all.

This last discussion, about the behavior of the DAT under high inhibitor number conditions, brings up one more issue that requires modification of the model. If the inhibitor completely binds to all transporter sites, the dopamine concentration will still decrease with time, through reactions with molecules other than DATs, and through diffusion. The apparent Michaelis constant can never, in practice, reach infinity, which Equation (5) would predict if $p_{\mathrm{o}}=1$. Thus, a term must be included in the model that keeps $K_{\mathrm{m}}(\mathrm{app})$ finite in the extreme case of very high inhibitor numbers. To implement this last important concept into the model, its final form becomes

$$
K_{\mathrm{m}}(\mathrm{app})=\left(\frac{1}{1-p_{\mathrm{o}}+b}\right) K_{\mathrm{m}} .
$$

The constant $b$ is a small number with a value around $\sim 0.005$. Physiologically, it accounts for dopamine diffusion and uptake in the presence of saturating amounts of cocaine, which means that the cocaine-responsive DATs are effectively absent (actually occupied by cocaine). Note that under cocaine-saturating conditions, the apparent Michaelis constant becomes $K_{\mathrm{m}} / b$. In the application of the final model, Equation (7), to the experimental data, there are three parameters whose values are varied to fit the model to the data. Two of the constants are: (1) $s(0)$, the initial cocaine-to-transporter number ratio at the time of an injection, ie at time $t=0$, when cocaine first floods the probed region; and (2) the constant $b$. Note that the initial inhibitor-occupied-transporter probability $p_{\mathrm{o}}(0)$ is directly related to $s(0)$. The third parameter needed is the decay rate $k_{\text {decay }}$ of the inhibitor number (or concentration) [I] after an injection of cocaine. In this case it is assumed that $s$, which is proportional to [I], decays exponentially in time after an inhibitor (cocaine) injection.

To explicitly describe the detailed time dependence of the model as it is used to fit the experimental data (see Figure 3), consider a set of data that has cocaine injections at times $t_{0}, t_{1}, t_{2} \ldots$ This means that the inhibitor number [I], and therefore $s$, during the whole time series has the form

$$
s(t)=s(0) \sum_{i=1}^{N} \mathrm{e}^{-k_{\text {decay }}\left(t-t_{i}\right)}
$$

where $N$ is the total number of injections.

The model was used on the data from naive rats repeatedly receiving a dose of cocaine $(0.75 \mathrm{mg} / \mathrm{kg})$, as described in the previous section and is shown in Figure 3. The model fits these data extremely well with the best-fit values being: $s(0)=3.83, b=0.0047$, and $k_{\text {decay }}=0.0628$ / min. The reduced $\chi^{2}$ for this fit is $\chi_{\mathrm{R}}=0.177$, with the number of degrees of freedom $=39$.

To definitively test the predictive capability and efficacy of the model, it was used to blindly predict the values of $K_{\mathrm{m}}$ (app) in the case where the inter-infusion intervals of the maintenance phase were made very short $(2 \mathrm{~min})$ and very long $(10 \mathrm{~min})$. Note these intervals do not correspond to inter-infusion intervals selected by rats during the selfadministration of cocaine, but were chosen outside this range to determine if the model would predict outcomes of future experiments. An experiment was then initiated for the long inter-infusion intervals with naive rats. The results demonstrated that the model can capture not only the important qualitative features of the dynamics of dopamine concentration, but it successfully described the outcome of the experiment (Figures 3 and 4). Note that no parameters were adjusted in generating the model points - these points were generated before voltammetric experiments based on the model's fit to rat responses in the case where the interinfusion intervals were much shorter ( $5 \mathrm{~min})$. 


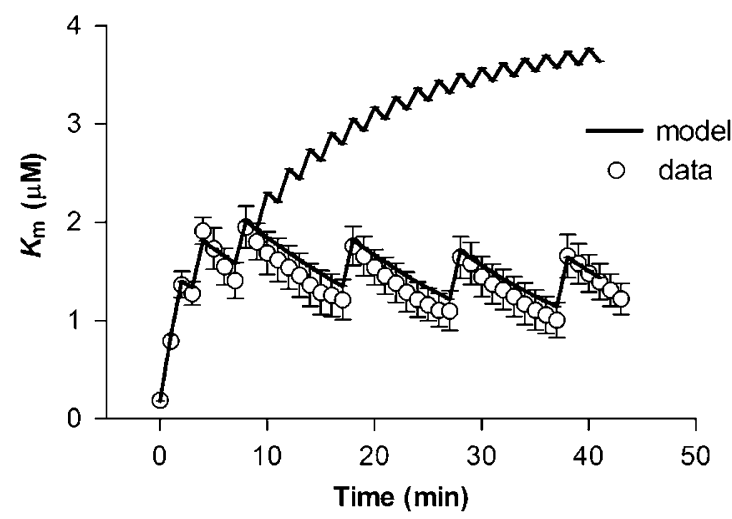

Figure 4 Changes in cocaine-induced dopamine uptake inhibition can be predicted. Two lines show predicted changes in dopamine uptake inhibition using two different inter-infusion intervals. The two curves correspond to blind predictions for the case where the inter-infusion intervals for the maintenance phase are $2 \mathrm{~min}$ apart (upper curve) and for an inter-infusion interval of $10 \mathrm{~min}$ (lower curve). Dopamine uptake changes using $10 \mathrm{~min}$ inter-infusion intervals, which were measured in animal experiments, are indicated by empty circles. Data are means \pm SEM of five rats per group. The experimental measurements agree well with the blind prediction from the model.

\section{DAT Inhibition Thresholds are Shifted Upward with Escalated Inter-Infusion Intervals (Experiment 4)}

Previous studies have demonstrated that providing long access to cocaine (6-h session) under a fixed-ratio 1 schedule for 14 days results in animals increasing, or 'escalating' the rate of cocaine intake across sessions (Ahmed and Koob, 1998, 1999). As illustrated in Figure 5, dopamine uptake inhibition $\left(K_{\mathrm{m}}(\mathrm{app})\right)$ was assessed while cocaine $(0.75 \mathrm{mg} / \mathrm{kg}$, i.v.) was infused into naive rats using inter-infusion intervals that were predetermined from rats that either escalated (long-access) or did not escalate (shortaccess) cocaine self-administration rates. The escalated intervals were determined to be $1.00,1.00,1.48,1.65,3.00$, $3.37,3.88,4.00,3.00,3.00,3.20,3.17,4.00$, and $3.12 \mathrm{~min}$ (see inter-infusion interval determinations in the Material and Methods). Similar to the previous experiment, cocaineinduced dopamine uptake inhibition (changes in $K_{\mathrm{m}}(\mathrm{app})$ ) appeared in two distinct phases, which correspond to the loading and maintenance phases observed during selfadministration under a fixed-ratio schedule (Ahmed and Koob, 1999; Wee et al, 2007; Specio et al, 2008). The total level of DAT inhibition was extensively higher with escalated (long-access) inter-infusion intervals, compared with that from unescalated (short-access) intervals (Figure 5). There was a significant main effect of the access condition from which rates were determined (ie long access $v s$ short access) on $K_{\mathrm{m}}$ (app) $(\mathrm{F}(1,336)=60.65 ; p<0.01)$. There was also a significant main effect of time $(\mathrm{F}(41,336)=5.45 ; p<0.01)$ on $K_{\mathrm{m}}(\mathrm{app})$, although the interaction of access condition and time was not statistically significant. Upper and lower thresholds were compared between escalated (long-access) and unescalated (shortaccess) groups by averaging the peaks and troughs of the oscillatory pattern of $K_{\mathrm{m}}(\mathrm{app})$ changes observed during the maintenance phase. For the escalated (long-access) rats the upper threshold was $2.81 \pm 0.03 \mu \mathrm{M}$ and the lower threshold

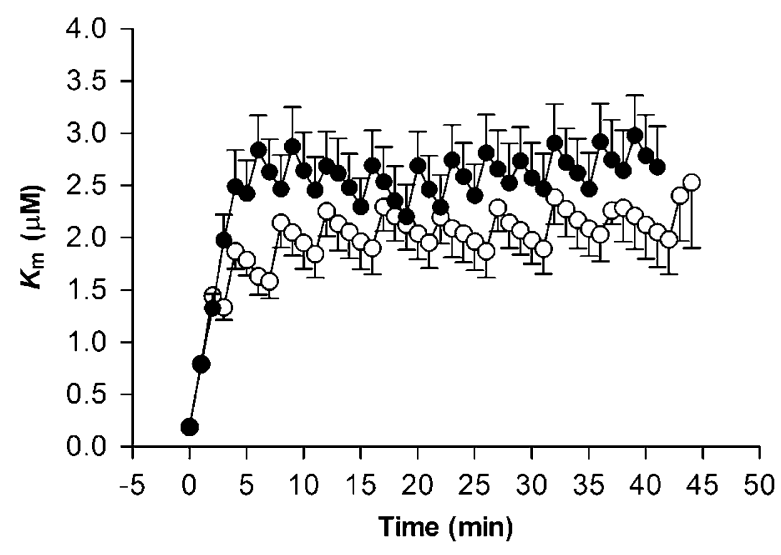

Figure 5 Dopamine transporter (DAT) inhibition thresholds are shifted upward during escalated cocaine self-administration. Dopamine uptake changes $\left(K_{m}(a p p)\right)$ obtained using inter-infusion intervals that were predetermined from rats with escalated rates of cocaine self-administration are indicated by solid black circles. Empty circles show cocaine-induced $K_{m}$ (app) alterations, which were observed with nonescalated intervals. All of the experiments displayed in this graph were conducted using cocaine naive rats. Data are means \pm SEM of five rats per group.

was $2.42 \pm 0.04 \mu \mathrm{M}(n=11)$. For the unescalated (shortaccess) rats the upper threshold was $2.28 \pm 0.03 \mu \mathrm{M}$ and the lower threshold was $1.94 \pm 0.03 \mu \mathrm{M}(n=6)$. There was a significant difference between the escalated (long-access) and unescalated (short-access) groups upper $(t=11.33$; $p<0.01)$ and lower $(t=8.976 ; p<0.01)$ thresholds.

\section{Escalated Cocaine Self-Administration Modifies the Maximal Rate of Dopamine Uptake (Experiment 5)}

In experiment 5 , the dopamine uptake parameters before and after four subsequent occuring cocaine $(0.75 \mathrm{mg} / \mathrm{kg}$, i.v.) infusions were compared between the cocaine naive group and rats that demonstrated escalated cocaine intake under a fixed-ratio 1 schedule (long-access training). The experiment indicated that the basal (predrug) value of $K_{\mathrm{m}}$ (app) was not different between cocaine-naive and cocaine-exposed rats $(0.18 \pm 0.008$ vs $0.18 \pm 0.008 \mu \mathrm{M}$; NS, $n=7)$. However, there was a significant difference in the basal $V_{\max }$ between these two groups $(t=4.249 ; p<0.01)$ (Figure 6a). As was expected (see results with single cocaine injection), acute cocaine infusions did not significantly alter this parameter of dopamine uptake in both cocaine-naive and drug-exposed animals (data not shown). Cocaineinduced $K_{\mathrm{m}}$ (app) changes were indistinguishable between these groups (Figure 6b). A two-way ANOVA demonstrated that the effect of prolonged cocaine exposure was not significant $(F(1,40)=0.043$; NS $)$, whereas the effect of acute cocaine was significant $(\mathrm{F}(4,40)=0.043 ; p<0.01)$.

\section{DISCUSSION}

The present study demonstrates that cocaine-induced DAT inhibition fluctuates over a time course that can account for patterns of cocaine self-administration reinforced under a fixed-ratio 1 schedule. Specifically, the inter-infusion intervals that are maintained during cocaine self-adminis- 

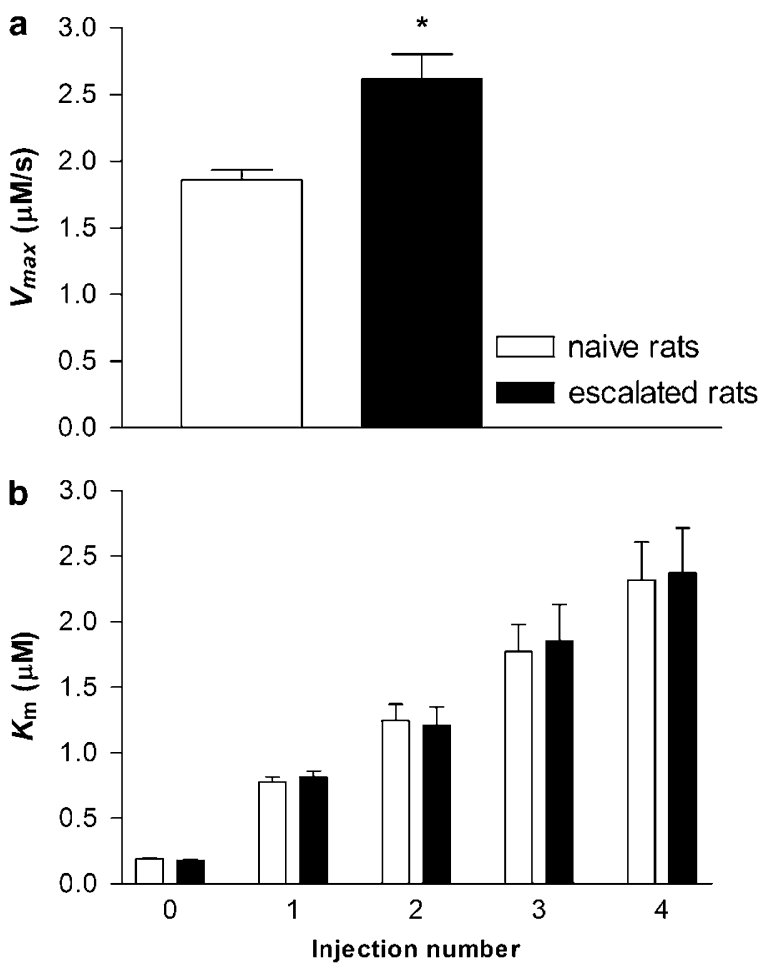

Figure 6 Consequences of escalated cocaine self-administration on the dopamine uptake in rat nucleus accumbens. (a) $V_{\max }$ is significantly increased following escalated cocaine intake (black bar) in comparison with naive control (empty bar). Data are means \pm SEM of seven rats per group. *** $<0.005$. (b) No significant changes in the affinity of dopamine transporter (DAT) for cocaine $\left(K_{m}(\mathrm{app})\right)$ are found in rats following escalation in cocaine self-administration (black bars) compared with naive control (empty bars). Four cocaine injections $(0.75 \mathrm{mg} / \mathrm{kg}$, i.v.) were performed to mimic loading phase. Data are means \pm SEM of five rats per group.

tration correlate with the maintenance of a rapidly changing level of DAT inhibition. These oscillating changes in dopamine uptake inhibition were modeled and can be predicted in a manner relevant to cocaine self-administration. Furthermore, this tightly maintained level of DAT inhibition was found to shift upward using intervals obtained after a history of escalated (long-access training) cocaine intake. Although, daily 6-h access (long access) during cocaine self-administration did not significantly alter the efficacy of cocaine for the DAT, it did result in a facilitated uptake of dopamine ( $V_{\max }$ changes) suggesting an upregulation of DAT number in response to a history of high cocaine intake.

\section{Single Intravenous Cocaine Administration and Uptake of Endogenous Dopamine}

The effect of i.v. injected cocaine on dopamine uptake occurs with a rapid onset and offset. The present data - together with previously published in vivo voltammetry data using i.p. cocaine administration ( $\mathrm{Wu}$ et al, 2001), as well as with in vitro drug application (Jones et al, 1995) - confirm that competitive inhibition of the DAT is the primary mechanism of acute cocaine action on increasing accumbal dopamine transmission. Indeed, i.v. cocaine dose dependently decreased the uptake of dopamine, acting through an alteration in $K_{\mathrm{m}}$ (app), whereas $V_{\max }$ remained unaffected. According to single curve analysis, the maximum effect of cocaine on dopamine uptake inhibition $\left(K_{\mathrm{m}}(\mathrm{app})\right)$ was reached within 1-2 min after a single cocaine infusion and then gradually decreased (Figure 1b). Importantly, the maximal effect of cocaine on $K_{\mathrm{m}}(\mathrm{app})$ is consistent with a peak in cocaine brain concentrations (Fowler et al, 1998; Ahmed et al, 2003) and the maximal inhibition of dopamine cells in the ventral tegmental area (Einhorn et al, 1988). These data should be contrasted to the results of Kiyatkin et al (2000) who demonstrated that cocaine-induced changes in clearance of exogenous dopamine began $2 \mathrm{~min}$ following a cocaine infusion, when extracellular dopamine concentrations were clearly elevated (Wise et al, 1995; Ahmed et al, 2003; Heien et al, 2005) and peaked at 6-8 min (Kiyatkin et al, 2000). Therefore, the dynamics of dopamine uptake inhibition following i.v. cocaine administration (see also Mateo et al, 2004; Samaha et al, 2004) appear to differ depending on whether stimulated dopamine release or exogenously applied dopamine is assessed.

\section{Levels of Dopamine Uptake Inhibition are Tightly Linked to Cocaine Self-Administration}

The present study shows a strong association between the pattern of cocaine self-administration and the level of dopamine uptake inhibition. Two stages of dopamine uptake inhibition are revealed in accordance with patterns of self-administration observed during cocaine self-administration under a fixed-ratio schedule. Previous reports, in addition to the data from the current study, have demonstrated that responding at the beginning of a selfadministration session (approximately $10 \mathrm{~min}$ ) occurs at a faster rate than at any other period of the session (Ahmed and Koob, 1999; Wee et al, 2007; Specio et al, 2008). This initial high rate of responding, termed the loading phase, is also reflected by a rapid increase in $K_{\mathrm{m}}(\mathrm{app})$ at the onset of a session (Figure 3). Following the loading phase, the rate of responding appears to subside and stabilize, presumably corresponding to the point in time at which an effective brain level of cocaine-induced DAT inhibition is reached. This level, which is reflected by a lower threshold of $K_{\mathrm{m}}(\mathrm{app})$, is then sustained through a maintenance phase during which responses are separated by stable interinfusion intervals (Ahmed and Koob, 1999; Specio et al, 2008). During the maintenance phase, the level of DAT blockade fluctuates around a narrow range within lower and upper thresholds.

The present data suggest that responding maintained by cocaine occurs in association with a lower threshold of dopamine uptake inhibition. This lower threshold has also been referred to as a trigger-point, set-point, or priming threshold (Wise et al, 1995; Ahmed and Koob, 1998; Tsibulsky and Norman, 1999). In fact, a lower level of extracellular dopamine within the nucleus accumbens has been previously implicated as a 'trigger' to respond during cocaine self-administration (Wise et al, 1995). The data from the current study link the 'trigger' to a lower threshold of dopamine uptake inhibition, thereby demonstrating the 
mechanism that provides the trigger-dopamine concentration.

The upper threshold has also received speculative attention (Pettit and Justice, 1989; Tsibulsky and Norman, 1999; Lynch and Carroll, 2001). For example, Pettit and Justice (1989) suggested that the upper threshold may be associated with aversive cocaine effects; whereas Tsibulsky and Norman (1999) suggested this threshold occurs because of satiety mechanisms alone. Although the data from the present study cannot reconcile why an upper threshold is observed, the observation that this threshold shifts upward when cocaine infusions are maintained at a high rate suggests that the upper threshold does not result from maximal DAT occupancy (see Figure 5).

Cocaine-induced dopamine uptake inhibition may alter phasic dopamine release through a $\mathrm{D}_{2}$ dopamine autoreceptor-mediated feedback mechanism (Grace, 2000; Phillips et al, 2003). It was discovered that a lever press for a cocaine infusion appears to be associated with subsecond dopamine release in the nucleus accumbens (Phillips et al, 2003; Stuber et al, 2005). Importantly, these short-lived dopamine changes - which may be influential in an animal's approach behavior toward a cocaine-paired lever or other drug-paired stimulus (Phillips et al, 2003; Stuber et al, 2005)-do not translate to a significant elevation in tonic dopamine levels. In contrast, DAT inhibition has a pronounced effect on tonic dopamine levels (Di Chiara and Imperato, 1988; Budygin et al, 2000; Heien et al, 2005). An intriguing speculation is that subsecond dopamine release can be promoted through feedback mechanisms resulting from a decrease in extrasynaptic dopamine as the level of cocaine-induced DAT inhibition approaches the lower threshold. Future studies are necessary to determine whether this connection exists.

\section{Consequences of Escalated Cocaine Self-Administration on Accumbal Dopamine Uptake}

Providing long access to cocaine during self-administration produces an increase, or 'escalation,' in the rate of cocaine self-administration (Ahmed and Koob, 1998), which is reflected by the maintenance of an increased level of dopamine uptake inhibition $\left(K_{\mathrm{m}}(\mathrm{app})\right)$. As illustrated in Figure 5 (experiment 4 ), the inter-infusion intervals obtained from animals with a history of long-access training were applied to determine the effect of an increased rate of self-administration on dopamine uptake inhibition in naive animals. It was found that cocaine-induced dopamine uptake inhibition reached a proportionally higher level during the loading phase consistent with an escalation of cocaine intake. Likewise, the dopamine uptake inhibition thresholds associated with the maintenance of responding during self-administration were shifted upward with 'escalated' intervals.

The neuroadaptations explaining the resulting changes in behavior during long-access training remain unknown. There is convincing evidence that changes in cocaine pharmacokinetics, and changes in baseline concentrations of dopamine in the nucleus accumbens, do not have a critical role in the escalation of cocaine intake observed during long-access training (Ahmed et al, 2003). Further- more, no apparent sensitization or desensitization to the effect of cocaine on accumbal dopamine concentrations was observed in animals with a history of long-access cocaine self-administration (Ahmed et al, 2003). However, it may be argued that prolonged cocaine exposure could modify the DAT inhibiting efficacy of cocaine. Examination of Figure 6b (experiment 5) shows that long-access escalation training did not result in significant changes in the efficacy $\left(K_{\mathrm{m}}(\mathrm{app})\right)$ of cocaine for the DAT. Therefore, the higher level of DAT blockade and subsequent maintenance of higher accumbal dopamine concentrations, which repeatedly take place during long-access cocaine self-administration, are not capable of significantly modifying the affinity of the DAT for cocaine. Thus, these data suggest that changes in the affinity of the DAT for cocaine are not involved in the escalation of cocaine intake.

Other possible neuroadaptations may occur during longaccess training, such as postsynaptic adaptations and an upregulation of DAT number, which may explain the escalation of cocaine intake. Several studies have reported postsynaptic adaptations following extended cocaine exposure demonstrating a downregulation of both striatal $\mathrm{D}_{1}$ (Graziella et al, 1998) and $\mathrm{D}_{2}$ (Nader et al, 2002) dopamine receptors. Assuming postsynaptic changes occur during an escalation of cocaine intake, it can be hypothesized that increased dopamine uptake inhibition thresholds are maintained to produce a level of reinforcement equal to that occurring before escalation. One of the possible consequences of prolonged increases in extracellular dopamine concentrations following administration of addictive substances, including cocaine, is an increase in the maximal rate of dopamine uptake (Budygin et al, 2003, 2007; Mateo et al, 2005). The data presented here demonstrate the existence of enhanced dopamine uptake in the rat nucleus accumbens after a history of long-access during cocaine self-administration (Figure 6a). The increased rate of dopamine uptake is likely the result of an upregulation in functional DAT number. Previous studies have reported increases in DAT availability following prolonged cocaine self-administration in both rats (Tella et al, 1996; but see also Ben Shahar et al, 2006) and nonhuman primates (Letchworth et al, 2001). Moreover, human post-mortem analyses report an increase in striatal DAT binding sites in cocaine addicts (Little et al, 1998, 1999). These presynaptic changes, which may occur following any regimen of prolonged cocaine exposure, may be a compensatory response of the dopamine system to persistently elevated dopamine concentration in the extrasynaptic space.

\section{CONCLUSION}

This study has implications for understanding the role of the DAT in regulating responding for cocaine during selfadministration. The present study took advantage of fastscan cyclic voltammetry, which provides high temporal and spatial resolution, and clearly demonstrates that a tight correlation exists between the level of dopamine uptake inhibition and the rate of responding for cocaine under a fixed-ratio schedule. 


\section{ACKNOWLEDGEMENTS}

We thank Dr Caroline Bass and Dr Jack Strandhoy for helpful comments and the UNC Department of Chemistry Electronics Facility for technical support. This work was supported by Wake Forest University Cross-Campus Collaborative Fund Award and National Institutes of Health grants DA021634 (EAB), F31DA024525 (EBO), and R01DA14030 (DCSR).

\section{DISCLOSURE/CONFLICT OF INTEREST}

The authors have no related financial interests or considerations to disclose.

\section{REFERENCES}

Ahmed SH, Koob GF (1998). Transition from moderate to excessive drug intake: change in hedonic set point. Science 282: 298-300.

Ahmed SH, Koob GF (1999). Long-lasting increase in the set point for cocaine self-administration after escalation in rats. Psychopharmacology (Berl) 146: 303-312.

Ahmed SH, Lin D, Koob GF, Parsons LH (2003). Escalation of cocaine self-administration does not depend on altered cocaineinduced nucleus accumbens dopamine levels. J Neurochem 86: 102-113.

Ben Shahar O, Moscarello JM, Ettenberg A (2006). One hour, but not six hours, of daily access to self-administered cocaine results in elevated levels of the dopamine transporter. Brain Res 1095: 148-153.

Budygin EA (2007). Dopamine uptake inhibition is positively correlated with cocaine-induced stereotyped behavior. Neurosci Lett 429: 55-58.

Budygin EA, John CE, Mateo Y, Daunais JB, Friedman DP, Grant KA et al (2003). Chronic ethanol exposure alters presynaptic dopamine function in the striatum of monkeys: a preliminary study. Synapse 50: 266-268.

Budygin EA, Kilpatrick MR, Gainetdinov RR, Wightman RM (2000). Correlation between behavior and extracellular dopamine levels in rat striatum: comparison of microdialysis and fast-scan cyclic voltammetry. Neurosci Lett 281: 9-12.

Budygin EA, Oleson EB, Mathews TA, Lack AK, Diaz MR, McCool BA et al (2007). Effects of chronic alcohol exposure on dopamine uptake in rat nucleus accumbens and caudate putamen. Psychopharmacology (Berl) 193: 495-501.

Di Chiara G, Imperato A (1988). Drugs abused by humans preferentially increase synaptic dopamine concentrations in the mesolimbic system of freely moving rats. Proc Natl Acad Sci USA 85: 5274-5278.

Einhorn LC, Johansen PA, White FJ (1988). Electrophysiological effects of cocaine in the mesoaccumbens dopamine system: studies in the ventral tegmental area. J Neurosci 8: 100-112.

Fowler JS, Volkow ND, Logan J, Gatley SJ, Pappas N, King P et al (1998). Measuring dopamine transporter occupancy by cocaine in vivo: radiotracer considerations. Synapse 28: 111-116.

Garris PA, Budygin EA, Phillips PE, Venton BJ, Robinson DL, Bergstrom BP et al. (2003). A role for presynaptic mechanisms in the actions of nomifensine and haloperidol. Neuroscience 118: 819-829.

Garris PA, Rebec GV (2002). Modeling fast dopamine neurotransmission in the nucleus accumbens during behavior. Behav Brain Res 137: 47-63.

Gerber GJ, Wise RA (1989). Pharmacological regulation of intravenous cocaine and heroin self-administration in rats: a variable dose paradigm. Pharmacol Biochem Behav 32: 527-531.
Grace AA (2000). The tonic/phasic model of dopamine system regulation and its implications for understanding alcohol and psychostimulant craving. Addiction 95(Suppl 2): S119-S128.

Graziella DM, Co C, Dworkin SI, Smith JE (1998). Modifications of dopamine D1 receptor complex in rats self-administering cocaine. Eur J Pharmacol 362: 9-15.

Heien ML, Khan AS, Ariansen JL, Cheer JF, Phillips PE, Wassum $\mathrm{KM}$ et al (2005). Real-time measurement of dopamine fluctuations after cocaine in the brain of behaving rats. Proc Natl Acad Sci USA 102: 10023-10028.

Jones SR, Garris PA, Wightman RM (1995). Different effects of cocaine and nomifensine on dopamine uptake in the caudateputamen and nucleus accumbens. J Pharmacol Exp Ther 274: 396-403.

Kiyatkin EA, Kiyatkin DE, Rebec GV (2000). Phasic inhibition of dopamine uptake in nucleus accumbens induced by intravenous cocaine in freely behaving rats. Neuroscience 98: 729-741.

Koob GF, Bloom FE (1988). Cellular and molecular mechanisms of drug dependence. Science 242: 715-723.

Letchworth SR, Nader MA, Smith HR, Friedman DP, Porrino LJ (2001). Progression of changes in dopamine transporter binding site density as a result of cocaine self-administration in rhesus monkeys. J Neurosci 21: 2799-2807.

Little KY, McLaughlin DP, Zhang L, McFinton PR, Dalack GW, Cook Jr EH et al (1998). Brain dopamine transporter messenger RNA and binding sites in cocaine users: a postmortem study. Arch Gen Psychiatry 55: 793-799.

Little KY, Zhang L, Desmond T, Frey KA, Dalack GW, Cassin BJ (1999). Striatal dopaminergic abnormalities in human cocaine users. Am J Psychiatry 156: 238-245.

Lynch WJ, Carroll ME (2001). Regulation of drug intake. Exp Clin Psychopharmacol 9: 131-143.

Mateo Y, Budygin EA, Morgan D, Roberts DC, Jones SR (2004). Fast onset of dopamine uptake inhibition by intravenous cocaine. Eur J Neurosci 20: 2838-2842.

Mateo Y, Lack CM, Morgan D, Roberts DC, Jones SR (2005). Reduced dopamine terminal function and insensitivity to cocaine following cocaine binge self-administration and deprivation. Neuropsychopharmacology 30: 1455-1463.

Montague PR, McClure SM, Baldwin PR, Phillips PE, Budygin EA, Stuber GD et al (2004). Dynamic gain control of dopamine delivery in freely moving animals. $J$ Neurosci 24: 1754-1759.

Nader MA, Daunais JB, Moore T, Nader SH, Moore RJ, Smith HR et al (2002). Effects of cocaine self-administration on striatal dopamine systems in rhesus monkeys: initial and chronic exposure. Neuropsychopharmacology 27: 35-46.

Near JA, Bigelow JC, Wightman RM (1988). Comparison of uptake of dopamine in rat striatal chopped tissue and synaptosomes. J Pharmacol Exp Ther 245: 921-927.

Pettit HO, Justice Jr JB (1989). Dopamine in the nucleus accumbens during cocaine self-administration as studied by in vivo microdialysis. Pharmacol Biochem Behav 34: 899-904.

Phillips PE, Stuber GD, Heien ML, Wightman RM, Carelli RM (2003). Subsecond dopamine release promotes cocaine seeking. Nature 422: 614-618.

Pickens R, Thompson T (1968). Cocaine-reinforced behavior in rats: effects of reinforcement magnitude and fixed-ratio size. J Pharmacol Exp Ther 161: 122-129.

Roberts DCS, Goeders NE (1989). Drug self-administration: experimental methods and determinants. In: Boulton AA, Baker GB, and Greenshaw AJ (eds). Neuromethods: Psychopharmacology. Humana Press: Clifton. pp 349-398.

Sabeti J, Gerhardt GA, Zahniser NR (2002). Acute cocaine differentially alters accumbens and striatal dopamine clearance in low and high cocaine locomotor responders: behavioral and electrochemical recordings in freely moving rats. J Pharmacol Exp Ther 302: 1201-1211. 
Sabeti J, Gerhardt GA, Zahniser NR (2003). Chloral hydrate and ethanol, but not urethane, alter the clearance of exogenous dopamine recorded by chronoamperometry in striatum of unrestrained rats. Neurosci Lett 343: 9-12.

Samaha AN, Mallet N, Ferguson SM, Gonon F, Robinson TE (2004). The rate of cocaine administration alters gene regulation and behavioral plasticity: implications for addiction. $J$ Neurosci 24: $6362-6370$.

Schmitz Y, Lee CJ, Schmauss C, Gonon F, Sulzer D (2001). Amphetamine distorts stimulation-dependent dopamine overflow: effects on D2 autoreceptors, transporters, and synaptic vesicle stores. J Neurosci 21: 5916-5924.

Schmitz Y, Schmauss C, Sulzer D (2002). Altered dopamine release and uptake kinetics in mice lacking D2 receptors. J Neurosci 22: 8002-8009.

Specio SE, Wee S, O'Dell LE, Boutrel B, Zorrilla EP, Koob GF (2008). CRF(1) receptor antagonists attenuate escalated cocaine self-administration in rats. Psychopharmacology (Berl) 196: 473-482.

Stuber GD, Wightman RM, Carelli RM (2005). Extinction of cocaine self-administration reveals functionally and temporally distinct dopaminergic signals in the nucleus accumbens. Neuron 46: 661-669.

Tella SR, Ladenheim B, Andrews AM, Goldberg SR, Cadet JL (1996). Differential reinforcing effects of cocaine and GBR12909: biochemical evidence for divergent neuroadaptive changes in the mesolimbic dopaminergic system. J Neurosci 16: 7416-7427.

Tsibulsky VL, Norman AB (1999). Satiety threshold: a quantitative model of maintained cocaine self-administration. Brain Res 839: 85-93.

Venton BJ, Seipel AT, Phillips PE, Wetsel BC, Gitler D, Greengard P et al. (2006). Cocaine increases dopamine release by mobilization of a synapsin-dependent reserve pool. J Neurosci 26: 3206-3209.
Voet D, Voet JG (2004). Biochemistry. Wiley: New York.

Volkow ND, Fowler JS, Wang GJ (2004). The addicted human brain viewed in the light of imaging studies: brain circuits and treatment strategies. Neuropharmacology 47(Suppl 1): 3-13.

Wakazono Y, Kiyatkin EA (2008). Electrophysiological evaluation of the time-course of dopamine uptake inhibition induced by intravenous cocaine at a reinforcing dose. Neuroscience 151: 824-835.

Wee S, Specio SE, Koob GF (2007). Effects of dose and session duration on cocaine self-administration in rats. J Pharmacol Exp Ther 320: 1134-1143.

Wightman RM, May LJ, Michael AC (1988). Detection of dopamine dynamics in the brain. Anal Chem 60: 769A-779A.

Wightman RM, Zimmerman JB (1990). Control of dopamine extracellular concentration in rat striatum by impulse flow and uptake. Brain Res Brain Res Rev 15: 135-144.

Wilson MC, Hitomi M, Schuster CR (1971). Psychomotor stimulant self administration as a function of dosage per injection in the rhesus monkey. Psychopharmacologia 22: 271-281.

Wise RA, Newton P, Leeb K, Burnette B, Pocock D, Justice Jr JB (1995). Fluctuations in nucleus accumbens dopamine concentration during intravenous cocaine self-administration in rats. Psychopharmacology (Berl) 120: 10-20.

Wu Q, Reith ME, Walker QD, Kuhn CM, Carroll FI, Garris PA (2002). Concurrent autoreceptor-mediated control of dopamine release and uptake during neurotransmission: an in vivo voltammetric study. J Neurosci 22: 6272-6281.

Wu Q, Reith ME, Wightman RM, Kawagoe KT, Garris PA (2001). Determination of release and uptake parameters from electrically evoked dopamine dynamics measured by real-time voltammetry. J Neurosci Methods 112: 119-133.

Yokel RA, Piekens R (1974). Drug level of D- and L-amphetamine during intravenous self-administration. Psychopharmacologia 34: 255-264. 